\title{
Erratum to: Environmental impacts of hybrid, plug-in hybrid, and battery electric vehicles - what can we learn from life cycle assessment?
}

\author{
Anders Nordelöf $^{1}$ - Maarten Messagie ${ }^{2}$ Anne-Marie Tillman ${ }^{1}$. \\ Maria Ljunggren Söderman $^{1}$ • Joeri Van Mierlo ${ }^{2}$
}

Published online: 26 November 2015

(C) Springer-Verlag Berlin Heidelberg 2015

\section{Erratum to: International Journal of Life Cycle Assessment DOI 10.1007/s11367-014-0788-0}

This is a corrigendum and clarification on behalf of the authors. Figure 4 in the original version of this article (Nordelöf et al. 2014a) has been corrected as regards the results for the Nissan Leaf BEV. The corrected Fig. 4 is presented below, calculated using the 2008 EU-mix electricity causing $467 \mathrm{~g} \mathrm{CO}_{2} / \mathrm{kWh}$ (Maas 2013) for the well-to-wheels life cycle, as intended and stated in the original article. The previous Fig. 4 incorrectly showed results based on a Belgian electricity mix. A related clarification, and correction, is made in section K.2, "Calculation of BEV results", in the Supplementary Information (Nordelöf et al. 2014b), where the last sentence of the last paragraph of the section should state: "Values can be approximated to $18 \mathrm{~g} \mathrm{CO}_{2}$-eq $/ \mathrm{km}$ for the net equipment life cycle, after recycling has been credited, and $79 \mathrm{~g} \mathrm{CO}_{2}$-eq $/ \mathrm{km}$ for the WTW life cycle based on the WTT stage."

The online version of the original article can be found at http://dx.doi.org/ 10.1007/s11367-014-0788-0.

Anders Nordelöf

anders.nordelof@chalmers.se

1 Department of Energy and Environment, Division of Environmental Systems Analysis, Chalmers University of Technology, 412 96, Gothenburg, Sweden

2 Research Centre MOBI-Mobility, Logistics and Automotive Technology Research Centre, Department of Electric Engineering and Energy Technology, Vrije Universiteit Brussel, Pleinlaan 2, 1050 Brussels, Belgium
Additionally, for clarification as regards Table 4 of the original article, the first sentence of the table caption should state: "Sensitivity of exemplified production related equipment life cycle GHG emissions to the lifetime driven distance, when presented per kilometer." Correspondingly, the following two sentences should be added to the second paragraph of section F, "Explanation of Table 4", of the Supplementary Information (Nordelöf et al. 2014b): "The data from the three studies has been selected and extracted at different levels of aggregation, and implies no harmonization of system boundaries between the examples. For full details about the equipment life cycle results of each study, we refer to the original references."

Finally, and most importantly, neither the corrections related to Fig. 4, nor the clarifications related to Table 4, alter any of the discussion or conclusions presented in the original article. 


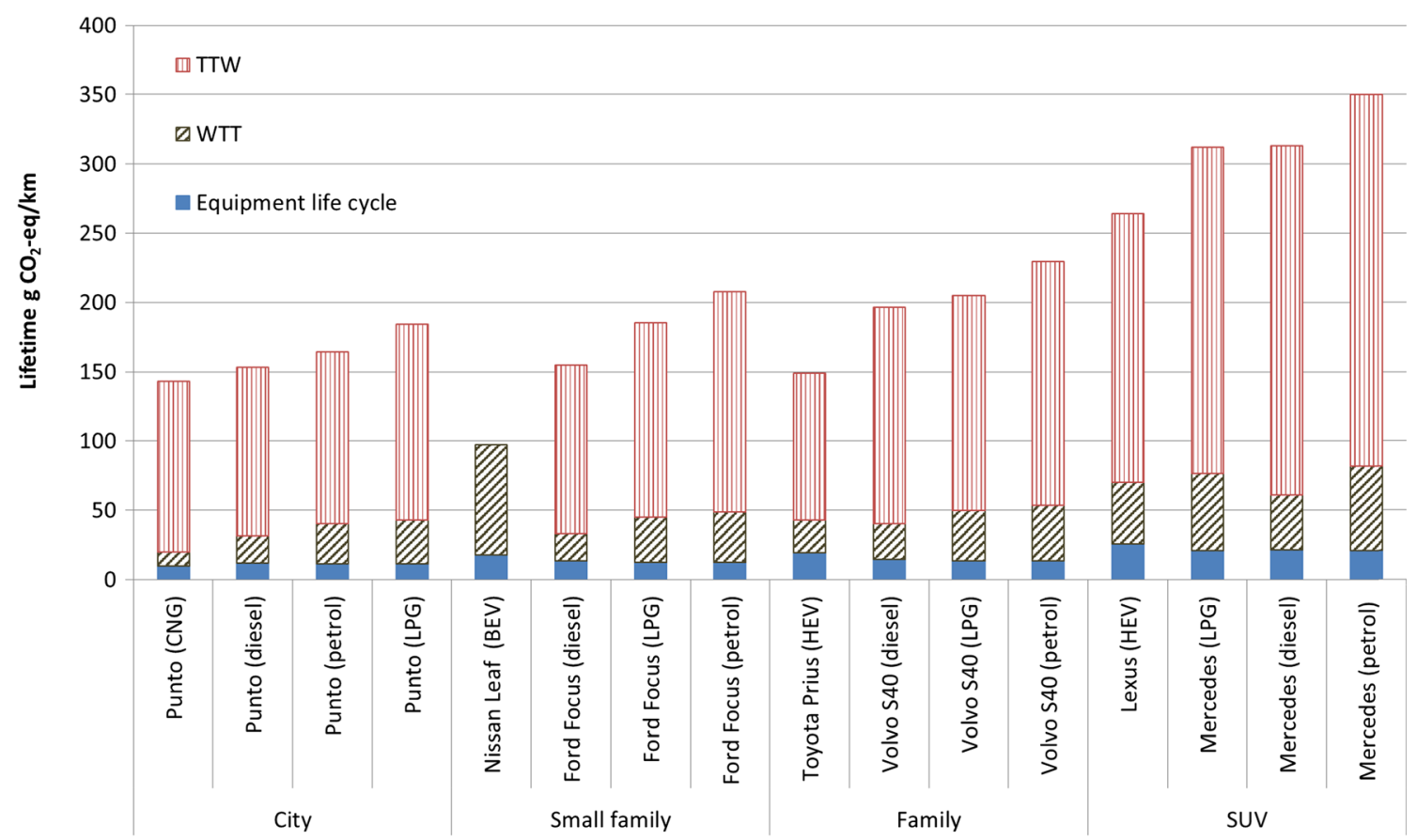

Fig. 4 Passenger cars divided into typical segments (Belgian Ecoscore classification) showing the general trend in $\mathrm{CO}_{2}$-emissions for the complete life cycle divided into WTT, TTW, and equipment life cycle based on the CLEVER study (Van Mierlo et al. 2009). An average vehicle lifetime of 230,500 km corresponding to 13.7 years has been used, based

on statistical data from the Belgian vehicle registration database. Fuel consumption is based on NEDC data. The Nissan Leaf BEV has been assumed to be charged with EU-mix electricity. For detailed information, see Electronic Supplementary Material

\section{References}

Maas H (2013) AW: Inquiry regarding calculations made in Edwards et al. 2011 WTW study Appendix 2. E-mail sent to Nordelöf A. Tuesday 23rd of April at 16:57, 2013

Nordelöf A, Messagie M, Tillman A-M, Ljunggren Söderman M, Van Mierlo J (2014a) Environmental impacts of hybrid, plug-in hybrid, and battery electric vehicles - what can we learn from life cycle assessment? Int J Life Cycle Assess 19(11):1866-1890. doi:10. 1007/s11367-014-0788-0

Nordelöf A, Messagie M, Tillman A-M, Ljunggren Söderman M, Van Mierlo J (2014b) Supplementary Information to the man-

uscript entitled Environmental impacts of hybrid, plug-in hybrid, and battery electric vehicles - what can we learn from life cycle assessment? Chalmers University of Technology, Sweden (Supplementary Information for International Journal of Life Cycle Assessment)

Van Mierlo J, Boureima F, Sergeant N, Wynen V, Messagie M, Govaerts L, Denys T, Vanderschaeghe M, Macharis C, Turcksin L, Hecq W, Englert M, Lecrombs F, Klopfert F, De Caevel B, De Vos M (2009) Clean vehicle research: LCA and policy measures "CLEVER"-science for a sustainable development-transport \& mobility, final report phase 1. Belgian Science Policy, Brussels (Research Programme Science for a Sustainable Development report SD/TM/04A) 